\title{
Diversidade da malacofauna associada à distribuição de Anomalocardia flexuosa (Linnaeus, 1767) (Mollusca: Bivalvia) em uma praia arenosa tropical
}

\author{
Severino Adriano de Oliveira Lima ${ }^{1 *}$ \\ Ana Júlia Rufino de Freitas ${ }^{2}$ \\ Humber Agrelli Andrade ${ }^{2}$ \\ ${ }^{1}$ Universidade Federal de Rondônia, Departamento de Engenharia de Pesca \\ Rua da Paz, 4376, Lino Alves Teixeira, CEP 76.916-000, Presidente Médici - RO, Brasil \\ ${ }^{2}$ Universidade Federal Rural de Pernambuco, Departamento de Pesca e Aquicultura \\ Rua Dom Manoel de Medeiros, Dois Irmãos, s/n, CEP 52.171-900, Recife - PE, Brasil \\ * Autor para correspondência \\ limasao@unir.com
}

Submetido em 29/04/2019

Aceito para publicação em 04/09/2019

\section{Resumo}

O conhecimento sobre a diversidade biológica é fundamental para o estabelecimento de medidas que conduzam à proteção de recursos naturais. As praias arenosas são áreas de alta diversidade, sendo a malacofauna um componente importante. Vários moluscos têm grande importância econômica e sua distribuição espacial, em praias arenosas, está atrelada a fatores físicos e biológicos. O objetivo deste estudo foi analisar a diversidade da malacofauna associada à distribuição de Anomalocardia flexuosa em uma praia arenosa tropical. As amostras foram coletadas bimensalmente, entre abril de 2016 e fevereiro de 2018, em 34 pontos distribuídos ao longo de todo o banco de areia. Foram calculadas as frequências de ocorrência das espécies e os índices de diversidade foram estimados e correlacionados com as densidades de A. flexuosa. No total foram obtidos 4.780 indivíduos, pertencentes a 11 espécies. As espécies mais frequentes foram A. flexuosa e Neritina virginea. Há correlações significativas entre as densidades de A. fleuxosa com as medidas de diversidade ocorrendo sobreposição entre os moluscos ao longo dos meses e dos locais de coleta. Portanto, as capturas dirigidas para essa espécie devem também considerar todos os outros moluscos para mitigar futuros danos à diversidade da malacofauna na região.

Palavras-chave: Bivalve; Diversidade; Ecologia quantitativa; Macrofauna

\section{Abstract}

Malacofauna diversity associated with distribution of Anomalocardia flexuosa (Linnaeus, 1767) (Mollusca: Bivalvia) on a tropical sandy beach. Knowledge about biological diversity is essential for the establishment of measures that lead to the protection of natural resources. Sandy beaches are areas of high diversity, with the malacofauna being an important component. Several mollusks are of great economic importance and the spatial distribution of these species on sandy beaches is linked to physical and biological factors. The aim of this study was to analyze the diversity of the malacofauna associated with Anomalocardia flexuosa distribution on a tropical sandy beach. Samples were collected bimonthly between April 2016 and 
February 2018 at 34 points distributed throughout a sandbar. The frequencies of occurrence of the species were calculated, and diversity indices were estimated and correlated with the densities of $A$. flexuosa. A total of 4,780 individuals from 11 species were obtained. The most frequent species were A. flexuosa and Neritina virginea. There were significant correlations between A. fleuxosa density and diversity, and overlap occurred between mollusks over the collection months and sites. Therefore, targeted catches for this species should also consider all other mollusks to mitigate future damage to malacofauna diversity in the region.

Key words: Bivalve; Diversity; Macrofauna; Quantitative ecology

\section{Introdução}

O conhecimento sobre a diversidade biológica é uma das questões básicas para o estabelecimento de medidas de proteção. As iniciativas correlacionadas à diversidade abrangem diferentes tipos de avaliações, porém o inventário de organismos vivos em uma determinada área é primordial (AMARAL; JABLONSKI, 2005). Áreas de alta diversidade incluem praias arenosas (DEXTER, 1992), onde as espécies da macrofauna bentônica são componentes importantes.

A macrofauna bentônica desempenha um importante papel ecológico no ciclo dos nutrientes, contribuindo para remineralização e produção primária em ambientes aquáticos (HENSEN et al., 2006). Entre os organismos bentônicos, os moluscos apresentam grande destaque nas praias arenosas explorando uma grande diversidade de habitat (e.g. epifauna e infaunal) e com variados hábitos alimentares (e.g. filtradores, raspadores) e, consequentemente, desempenhando um papel fundamental na ecologia da macrofauna desses ambientes (JARAMILLO et al., 2001). As espécies de moluscos coexistem em um mesmo local com muitos casos de sobreposição espacial (BEASLEY et al., 2005; MATTOS; CARDOSO, 2012; CRUZ-NETA; HENRYSILVA, 2013). No entanto, os bivalves são dominantes em termos de densidade e biomassa na macrofauna de praias abrigadas (MCLACHLAN; BROWN, 2006) e, nesse tipo de ambiente, tais espécies afetam a distribuição vertical e estabilidade dos sedimentos (HALL, 1994), os fluxos de nutrientes do sedimento para a coluna d'água (MICHAUD et al., 2006) e a disponibilidade de oxigênio por conta do hábito de escavação (MICHAUD et al., 2005).

O bivalve Veneridae Anomalocardia flexuosa ocorre nas Antilhas, em toda a extensão do litoral do Brasil (ABBOTT, 1974) e chega ao Uruguai (RIOS, 1994). No Brasil, a espécie é o componente dominante da macrofauna em vários locais (PEZZUTO; ECHTERNACHT, 1999; OLIVEIRA et al., 2011), porém, tem preferência por locais com águas calmas, sobretudo na presença de sedimento arenoso e principalmente areno-lodoso, onde pode se enterrar superficialmente no infralitoral raso e nas regiões entremarés (BOEHS et al., 2008). Pode ainda ocorrer em marismas e baixios não vegetados e com menor frequência em manguezais (SCHAEFFER-NOVELLI, 1980; BOEHS; MAGALHÃES, 2004).

A abundância e a distribuição espacial do bivalve A. flexuosa estão associadas a vários fatores como, por exemplo, salinidade (BARROSO; MATTHEWSCASCON, 2009), tipo de sedimento (BEASLEY et al., 2005), pluviosidade (OLIVEIRA et al., 2011), predação durante a fase larval (BORZONE et al., 1996; BOEHS; MAGALHÃES, 2004), entre outros. Há também associação com o padrão de distribuição desse bivalve com interações bióticas (MATTOS; CARDOSO, 2012), sendo que outras espécies da macrofauna também apresentam esse comportamento (DUGAN et al., 2004). Para o entendimento das variações de abundância de A. flexuosa é preciso avaliar a interação dessa espécie com outros moluscos, principalmente com bivalves de hábito alimentar similar e que podem ser potenciais competidores. Além disso, é importante compreender a dinâmica da malacofauna ao longo de todo banco arenoso que apresenta características distintas, principalmente em relação à influência marinha e de rios, e se há uma sazonalidade marcante nesse local. Portanto, o objetivo deste estudo foi analisar a diversidade da malacofauna associada à distribuição de $A$. flexuosa em uma praia arenosa tropical. 


\section{Material e Métodos}

\section{Área de estudo e estratos de localização}

O banco extrativista da praia de Mangue Seco está localizado no município de Igarassu, que dista $30 \mathrm{~km}$ da capital Recife, Pernambuco, Nordeste do Brasil. O banco se estende no sentido sul-norte, desde a foz do Rio Timbó até a foz sul do Canal de Santa Cruz, abrangendo uma área de aproximadamente $2,7 \mathrm{~km}^{2}$ (Figura 1). A praia tem aproximadamente $2 \mathrm{~km}$ de extensão, com águas rasas e pequenas ondas (OLIVEIRA et al., 2014).
O sedimento encontrado na praia é arenoso, composto por mais de $90 \%$ de areia, com predominância de areia grossa $(68,7 \%)$, e areia fina na proporção de $27,2 \%$ (LAVANDER et al., 2011). A salinidade da água no banco variou de 37 (janeiro) a 25 (junho); a temperatura máxima foi de $37^{\circ} \mathrm{C}$ em dezembro e mínima em fevereiro $\left(28^{\circ} \mathrm{C}\right)$ no ano de 2009 (LAVANDER et al., 2011).

Os estratos de localização foram definidos de acordo com o zoneamento proposto por Lima e Andrade (2018), com os pontos amostrais distribuídos de forma equidistante entre seis setores, em que foram considerados os efeitos de bordas nos contatos com os

FIGURA 1: Banco extrativista da praia de Mangue Seco, localizado no município de Igarassu (PE) com indicação dos 34 pontos de coleta estratificados em: borda mar (círculos azuis), borda rio (círculos amarelos), interior costa (círculos vermelhos) e interior mar (círculos brancos).

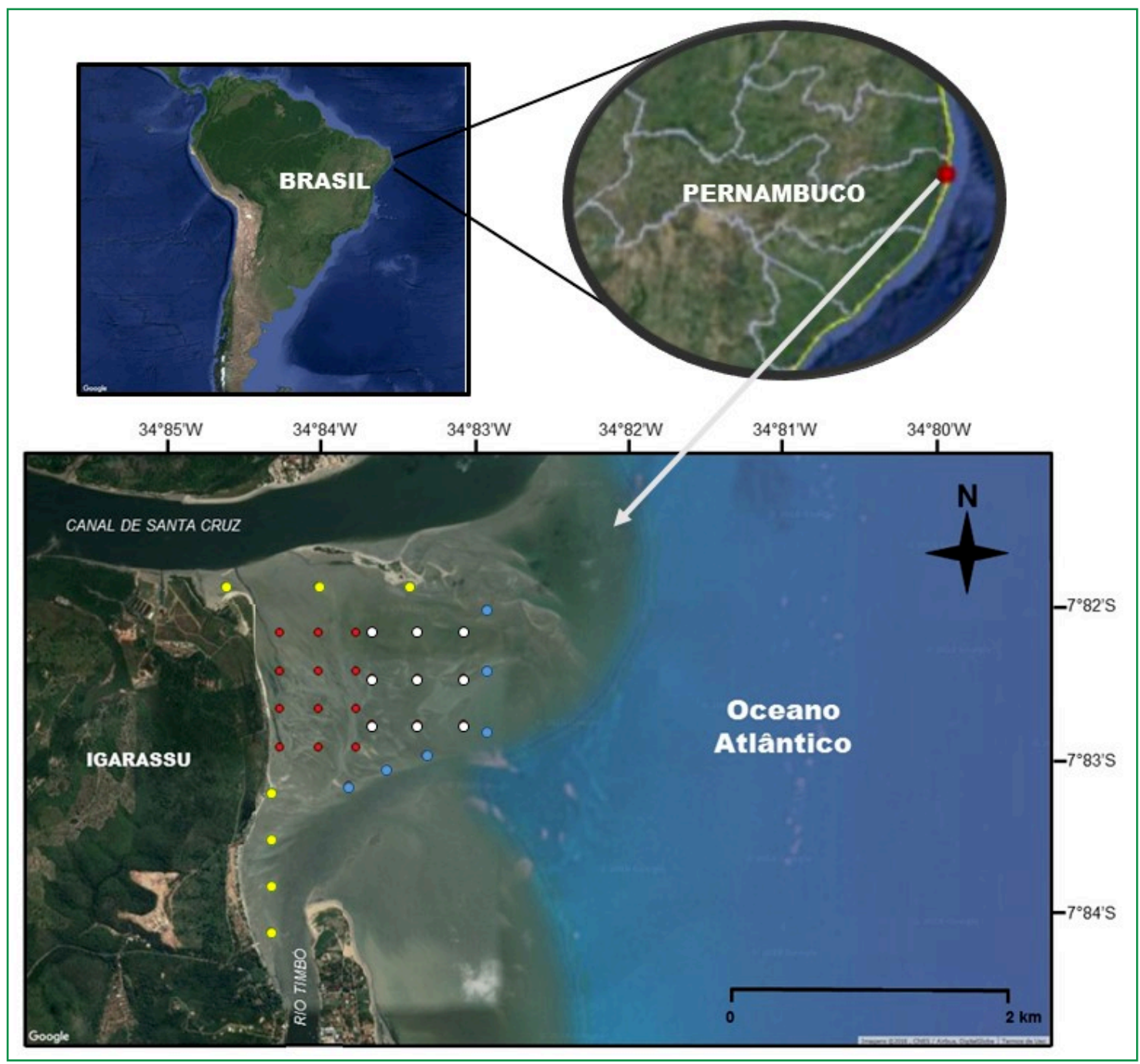


corpos de água circundantes e a proximidade da linha de praia, e reduzidos para quatro estratos, como sugerido pelos autores e apresentado na Figura 1. Assim, esses quatro estratos foram avaliados e denominados de borda mar (BM), borda rio (BR), interior costa (IC) e interior mar (IM). As bordas do banco correspondem às regiões mais expostas à ação da água circundante, seja ela de origem marinha (BM) ou fluvial (BR), e, portanto, estão mais sujeitas à influência de ondas e correntes. $\mathrm{O}$ estrato IC corresponde a uma zona mais abrigada e menos exposta a ondas e correntezas. O estrato IM também é localizado no interior do banco, porém em posição mais intermediária entre a linha da praia e o mar e está menos exposto que as bordas à influência marinha e fluvial, no entanto mais exposto que a região costeira interna IC.

\section{Coleta dos dados}

As amostras foram coletadas bimensalmente no período entre abril de 2016 e fevereiro de 2018 em 34 pontos distribuídos ao longo de todo o banco de areia (Figura 1), sendo seis em BM, sete em BR, 12 em IC e nove em IM. O número de pontos alocados em cada estrato foi dependente do tamanho da área e da variabilidade das densidades de $A$. flexuosa em cada local (LIMA; ANDRADE, 2018). Cada campanha amostral incluiu três dias consecutivos de trabalho em maré baixa de sizígia para que se pudesse cobrir os 34 pontos amostrais. As amostras foram coletadas com um cilindro de policloreto de polivinila (PVC) de 19 $\mathrm{cm}$ de diâmetro que foi introduzido no sedimento a 10 $\mathrm{cm}$ de profundidade, onde o sedimento e organismos foram lavados com água, peneirados com malha quadrada de $1 \mathrm{~mm}$ entre nós adjacentes, contabilizados e identificados posteriormente em laboratório com ajuda de bibliografia pertinente (WARMKE; ABBOTT, 1961; RIOS, 1994).

\section{Análise dos dados}

As frequências de ocorrência (FO\%) das espécies de moluscos amostrados foram calculadas para cada mês de coleta. Logo, 100\% de FO corresponde à presença nos 34 pontos amostrados. Cálculos de FO também foram estimados para os estratos de localização com a observação de todos os meses.
A relação entre o número de espécies e o número de amostras foi verificada com curvas de rarefação, sendo constatada através do método de acumulação de espécies (curva de acumulação) para determinar a qualidade amostral pela assíntota da curva.

As densidades de A. flexuosa foram estimadas nos diferentes meses de coleta e estratos de localização, assim como os índices de diversidade e densidades. Os cálculos de diversidade foram realizados excluindo-se A. flexuosa. Nos cálculos das densidades dos outros moluscos, foram também considerados os dados com todas as outras espécies agregadas e com apenas as espécies de bivalves.

Os índices de diversidade estimados foram os de Shannon-Weaver (SHANNON; WEAVER, 1949), dominância de Simpson (SIMPSON, 1949) e equitabilidade de Pielou (PIELOU, 1969), além da riqueza numérica (número de espécies em cada amostra). No índice de Shannon-Weaver frequentemente assume-se que os indivíduos são amostrados ao acaso a partir de uma população "indefinidamente grande" e que todas as espécies estão representadas, sendo relativamente independente do tamanho da amostra. O índice de Simpson exprime a abundância das espécies mais comuns, sendo, consequentemente, mais sensível às mudanças que ocorrem neste grupo de espécies em particular. É um índice de dominância e a derivação desse cálculo está relacionada à probabilidade de dois indivíduos escolhidos ao acaso na comunidade pertencerem à mesma espécie. Já o índice de Pielou expressa o quão igualmente os indivíduos estão distribuídos entre as diferentes espécies.

Nos cálculos da curva de rarefação e dos índices de diversidade, foi utilizado o pacote Vegan (OKSANEN et al., 2018). As medidas de diversidade e densidades estimadas nos diferentes meses e estratos espaciais não atenderam aos pressupostos para inferência paramétrica. Portanto, optou-se pelo uso do teste não paramétrico de Kruskal-Wallis $(\alpha=0,05)$ para as análises das densidades da A. flexuosa nos meses e estratos de localização, além das diversidades de Shannon-Weaver, Simpson e Pielou, também nos meses e estratos de localização. Nos casos de diferenças significativas, procedeu-se com um pósteste de comparações múltiplas. As correlações entre as 
densidades de A. flexuosa com os índices de diversidade e densidade dos outros moluscos foram avaliadas com o teste de correlação de Spearman $(\alpha=0,05)$. Todas as análises foram realizadas com o programa R (R CORE TEAM, 2018).

\section{Resultados}

No total, foram obtidos 4.780 indivíduos, sendo 3.306 da classe Bivalvia e 1.474 da classe Gastropoda. As espécies identificadas são apresentadas na Figura 2, sendo que Anomalocardia flexuosa (Veneridae) foi a que apresentou o maior número de indivíduos $(\mathrm{n}=3.218)$, seguida por Neritina virginea (Neritidae) $(\mathrm{n}=1.079)$. As outras espécies foram: Iphigenea brasiliensis (Donacidae) $(\mathrm{n}=13)$, Lucina pectinata (Lucinidae) $(\mathrm{n}=2)$, Macoma constricta, Tellina lineata, e T. versicolor (Tellinidae) ( $\mathrm{n}=5,20$ e 48, respectivamente), Bulla striata (Bullidae) $(\mathrm{n}=1)$, Cerithium atratum (Cerithiidae) $(\mathrm{n}=107)$, Nassarius polygonatus (Nassariidae) $(\mathrm{n}=18)$, Olivella petiolita (Olividae) $(\mathrm{n}=269)$.

A frequência de ocorrência de $A$. flexuosa foi sempre superior à de todos os moluscos ao longo dos meses (Tabela 1). A menor frequência para essa espécie foi em abril de 2016 e as maiores em agosto do mesmo ano e em abril de 2017. Entre os bivalves, com exceção de $A$. flexuosa, a que mais se destacou em termos de frequência ao longo do período foi $T$. versicolor; entre os gastrópodes tiveram destaque $N$. polygonatus, $N$. virginea e $O$. petiolita, respectivamente.

Nas diferentes regiões do banco a frequência de ocorrência (FO) de A. flexuosa também foi sempre superior à de todos os outros moluscos (Tabela 2). A frequência para essa espécie foi maior no estrato IC, seguido de IM, BR e BM, respectivamente. A maioria das espécies tiveram maior frequência no estrato IC, porém os bivalves $M$. constricta e $I$. brasiliensis, e principalmente o gastrópode $O$. petiolita, foram mais frequentes no estrato IM. Considerando todos os moluscos, exceto A. flexuosa, O. petiolita foi a que teve maiores valores de FO nas regiões BM e IM, enquanto $N$. virginea predominou em BR e IC.

A riqueza da malacofauna inventariada estimada com base em todas as amostras agregadas dos estratos de localização atingiu uma assíntota (Figura 3A), ou seja, a riqueza total observada $(\mathrm{n}=11)$ possivelmente não aumentaria (ou muito pouco) com a elevação do número de amostras. Nos estratos de localização separados as estimativas também atingiram uma assíntota (Figura 3B), com valores de riquezas observadas para BM $(n=6)$, BR $(n=8), \operatorname{IC}(n=11)$ e IM $(n=8)$.

Nas densidades de A. flexuosa ocorreram diferenças ao longo dos meses $\left(\chi^{2}=35,39, p=2,14 \times 10^{-4}\right.$, $\mathrm{gl}=11)$ e para as diferentes regiões do banco $\left(\chi^{2}=107,27\right.$, $p=2,20 \times 10^{-16}, \mathrm{gl}=3$ ) (Figura 4). As densidades dos meses de junho de 2016 e abril, junho e agosto de 2017

FIGURA 2: Fotos das espécies identificadas no banco extrativista da praia de Mangue Seco (sem escala). Bivalves: 1. Anomalocardia flexuosa; 2. Iphigenia brasiliensis; 3. Lucina pectinata; 4. Macoma constricta; 5. Tellina lineata; 6. Telllina versicolor; Gastrópodes: 7. Bulla striata; 8. Cerithium atratum; 9. Nassarius polygonatus; 10. Neritina Virgenia; 11. Olivella petiolita.

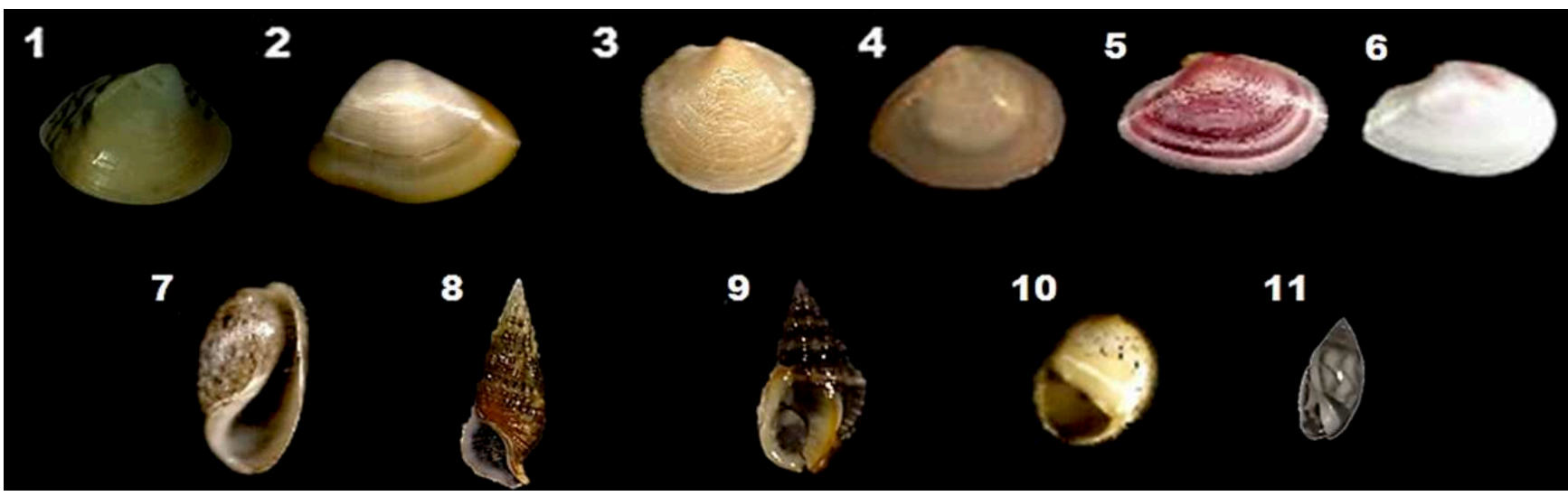


foram maiores do que as dos meses de abril e dezembro de 2016 e de fevereiro e dezembro de 2017 (Figura 4A). Nos estratos, as densidades em IC foram maiores do que nas demais localizações (Figura 4B). Os estratos BR e IM não apresentaram diferença entre si, mas as densidades foram maiores do que em BM.

TABELA 1: Frequência de ocorrência (\%) das espécies de moluscos identificadas no banco extrativista da praia de Mangue Seco de acordo com as 34 amostras realizadas ao longo dos meses.

\section{Espécie}

Anomalocardia flexuosa (Linnaeus, 1767)

Bulla striata Bruguière, 1792

Cerithium atratum (Born, 1778)

Iphigenia brasiliensis (Lamarck, 1818)

Lucina pectinata (Gmelin, 1791)

Macoma constricta (Bruguière, 1792)

Nassarius polygonatus (Lamarck, 1822)

Neritina virginea (Linnaeus, 1758)

Olivella petiolita (Duclos, 1835)

Tellina lineata W. Turton, 1819

Tellina versicolor Carpenter, 1864

\section{Mês}

abr/16 jun/16 ago/16 out/16 dez/16 fev/16 abr/17 jun/17 ago/17 out/17 dez/17 fev/18

\begin{tabular}{cccccccccccc}
50,00 & 70,59 & 79,41 & 73,53 & 64,71 & 73,53 & 79,41 & 76,47 & 73,53 & 70,59 & 67,65 & 73,53 \\
0,00 & 2,94 & 0,00 & 0,00 & 0,00 & 0,00 & 0,00 & 0,00 & 0,00 & 0,00 & 0,00 & 0,00 \\
5,88 & 0,00 & 0,00 & 8,82 & 8,82 & 8,82 & 11,76 & 2,94 & 2,94 & 2,94 & 2,94 & 2,94 \\
8,82 & 2,94 & 5,88 & 0,00 & 5,88 & 2,94 & 2,94 & 5,88 & 0,00 & 0,00 & 2,94 & 0,00 \\
0,00 & 2,94 & 2,94 & 0,00 & 0,00 & 0,00 & 0,00 & 0,00 & 0,00 & 0,00 & 0,00 & 0,00 \\
2,94 & 0,00 & 0,00 & 5,88 & 0,00 & 0,00 & 0,00 & 0,00 & 2,94 & 0,00 & 0,00 & 0,00 \\
2,94 & 5,88 & 8,82 & 5,88 & 5,88 & 5,88 & 2,94 & 2,94 & 2,94 & 2,94 & 0,00 & 2,94 \\
17,65 & 23,53 & 26,47 & 32,35 & 38,24 & 32,35 & 41,18 & 26,47 & 29,41 & 17,65 & 29,41 & 20,59 \\
17,65 & 17,65 & 20,59 & 32,35 & 35,29 & 29,41 & 29,41 & 29,41 & 26,47 & 14,71 & 20,59 & 20,59 \\
0,00 & 0,00 & 0,00 & 0,00 & 0,00 & 0,00 & 0,00 & 8,82 & 5,88 & 2,94 & 2,94 & 11,76 \\
5,88 & 23,53 & 2,94 & 2,94 & 2,94 & 2,94 & 5,88 & 11,76 & 14,71 & 11,76 & 0,00 & 8,82 \\
\hline
\end{tabular}

TABELA 2: Frequência de ocorrência (\%) das espécies de moluscos identificadas no banco extrativista da praia de Mangue Seco nas amostras realizadas por estrato de localização, agregados todos os meses.

\begin{tabular}{lcccc}
\hline \multirow{2}{*}{ Espécie } & \multicolumn{4}{c}{ Região do banco } \\
\cline { 2 - 5 } & Borda mar (BM) & Borda rio (BR) & Interior costa (IC) & Interior mar (IM) \\
\hline Anomalocardia flexuosa & 31,94 & 65,48 & 89,58 & 76,85 \\
Bulla striata & 0,00 & 0,00 & 0,69 & 0,00 \\
Cerithium atratum & 1,39 & 7,14 & 8,33 & 0,93 \\
Iphigenia brasiliensis & 2,78 & 1,19 & 3,47 & 4,63 \\
Lucina pectinata & 0,00 & 0,00 & 1,39 & 0,00 \\
Macoma constricta & 0,00 & 0,00 & 1,39 & 1,85 \\
Nassarius polygonatus & 2,78 & 3,57 & 5,56 & 3,70 \\
Neritina virginea & 2,78 & 20,24 & 52,08 & 18,52 \\
Olivella petiolita & 27,78 & 5,95 & 15,28 & 49,07 \\
Tellina lineata & 0,00 & 4,76 & 4,86 & 0,00 \\
Tellina versicolor & 2,78 & 9,52 & 13,89 & 1,85 \\
\hline
\end{tabular}


FIGURA 3: Curvas de rarefação da malacofauna capturada no banco extrativista da praia de Mangue Seco considerando todas as amostras (A) e separadas pelas regiões borda mar (BM), borda rio (BR), interior costa (IC) e interior mar (IM) (B).

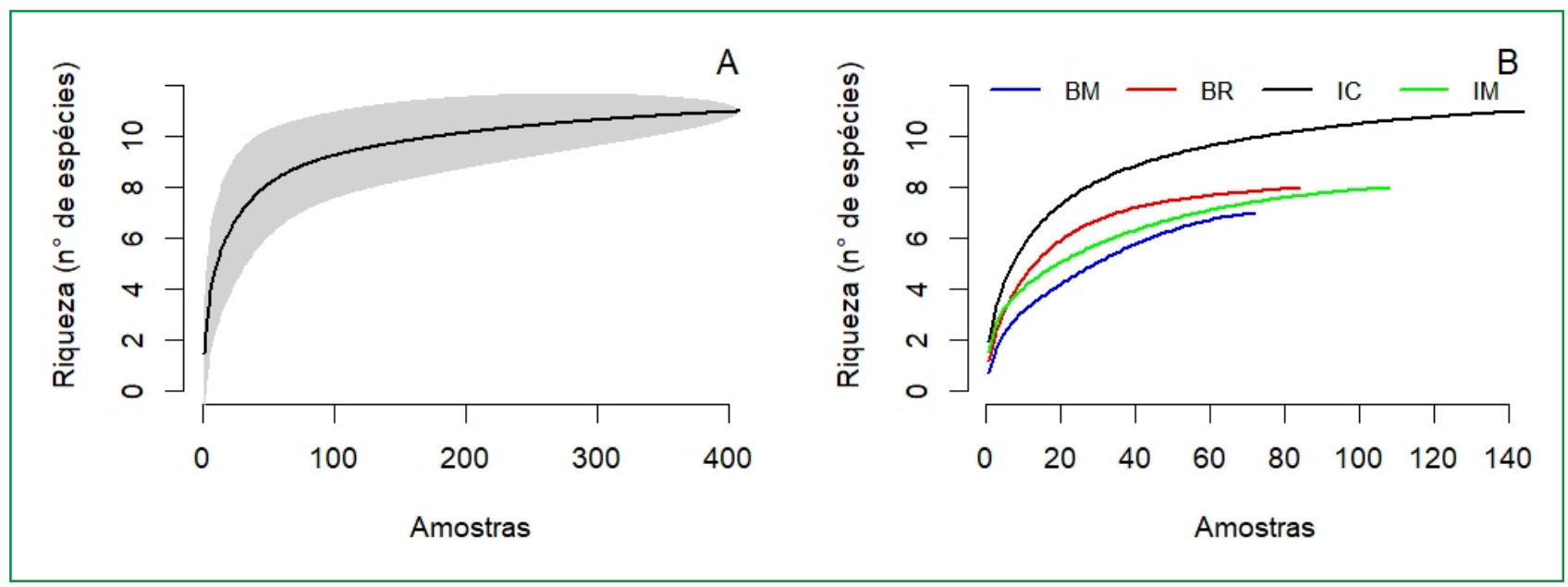

FIGURA 4: Diagramas de caixas com as densidades de Anomalocardia flexuosa no banco extrativista da praia de Mangue Seco ao longo dos meses de coleta (A) e por estrato de localização (B).

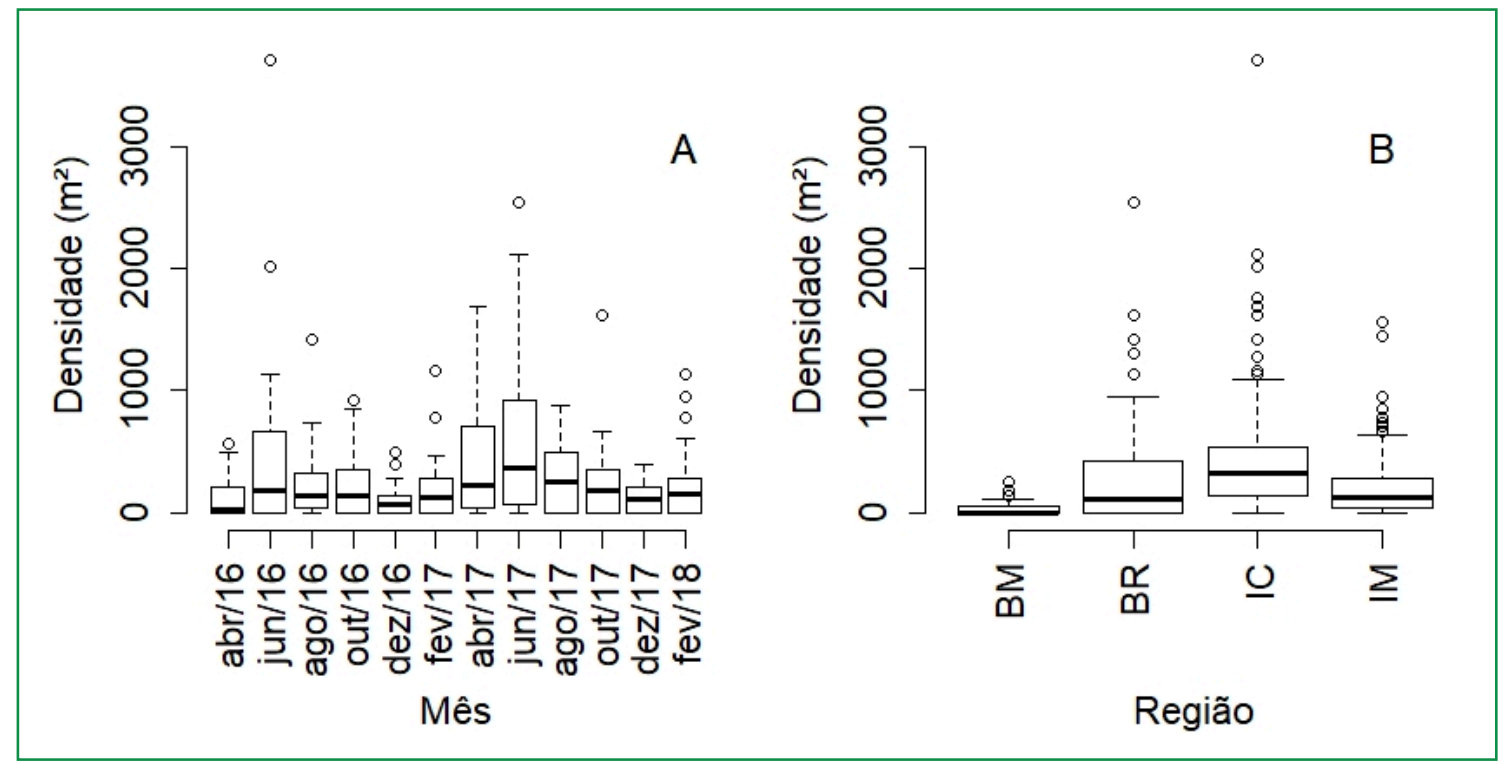

Não ocorreram diferenças ao longo dos meses para os valores do índice de Shannon-Weaver $\left(\chi^{2}=11,44, p=\right.$ $0,41, \mathrm{gl}=11)$, que tiveram todas as medianas igual a 0,00 e o máximo de 1,39 no mês de junho de 2017 (Figura $5 \mathrm{~A})$, nem para o índice de Simpson $\left(\chi^{2}=9,72, p=0,56\right.$, $\mathrm{gl}=11)$, no qual as maiores medianas ocorreram nos meses de abril e junho de 2016 e outubro e dezembro de $2017(1,00)$, e que também foram os valores máximos (Figura 5B), assim como para o número de espécies $\left(\chi^{2}=10,85, p=0,46, \mathrm{gl}=11\right)$, que teve maior mediana $(1,00)$ em sete dos 12 meses de coleta (outubro e dezembro de 2016, entre fevereiro de agosto de 2017 e em fevereiro de 2018), sendo o máximo de 4,00 em junho de 2017 (Figura 5C), como também para o índice de Pielou $\left(\chi^{2}=11,00, p=0,44, \mathrm{gl}=11\right)$, que teve valores de mediana de 0,00 para todos os meses e máximos de 1,00 também para todos os meses, exceto fevereiro de 2018 (Figura 5D). Assim como para as diversidades, as 
FIGURA 5: Diagramas de caixas com os índices de diversidade de Shannon (A), Simpson (B), Número de espécies (C), Pielou (D), Densidade de moluscos agregados (E), Densidade de bivalves agregados (F) da malacofauna capturada no banco extrativista da praia de Mangue Seco.

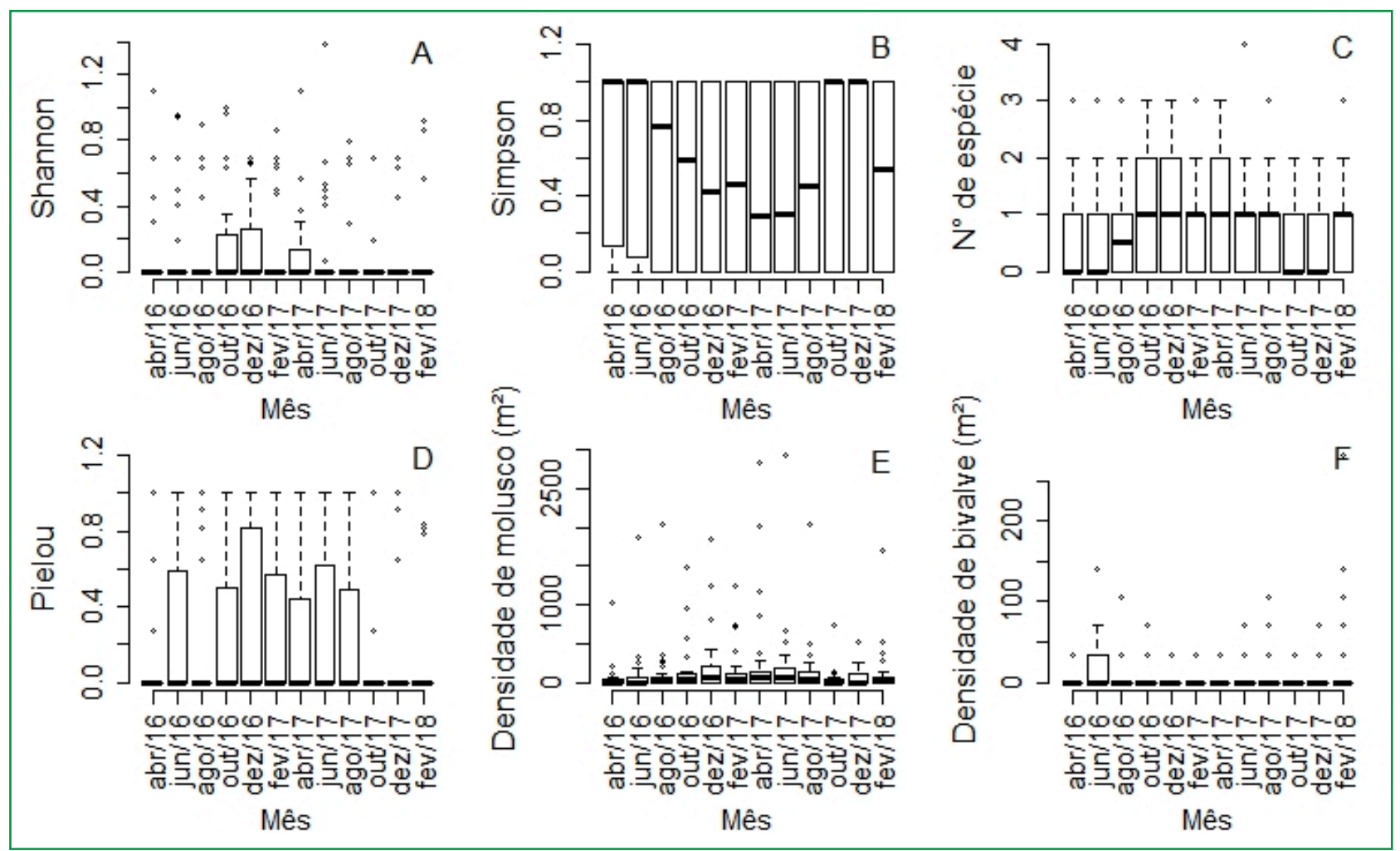

densidades dos moluscos ao longo dos meses também não apresentaram diferenças. Todos os moluscos agregados $\left(\chi^{2}=16,46, p=0,12, \mathrm{gl}=11\right)$ tiveram a maior mediana da densidade em junho de 2017 (71 ind. $\mathrm{m}^{-2}$ ) e o valor máximo de 2929 ind. m $^{-2}$ também ocorreu no mesmo mês. Já as densidades agregadas dos bivalves $\left(\chi^{2}=16,75, p=0,11, \mathrm{gl}=11\right)$ apresentaram todas as medianas com valor de 0 ind. $\mathrm{m}^{-2}$ e o máximo foi de 282 ind. $\mathrm{m}^{-2}$ em fevereiro de 2018 (Figura 5F).

Nos estratos de localização ocorreram diferenças para todos os índices de diversidade e densidades agregadas avaliadas (Figura 6). A mediana da região IC para o índice de Shannon $(0,18)$ foi maior do que os demais estratos (Figura 6A) $\left(\chi^{2}=14,76, p=2,00 \times 10^{-3}\right.$, $\mathrm{gl}=3$ ). Para o índice de Simpson (Figura 6B), as medianas $(1,00)$ das regiões de borda (BM e BR) foram maiores que as regiões de interior (IC e IM) $\left(\chi^{2}=33,80\right.$, $p=2,18 \times 10^{-7}, \mathrm{gl}=3$ ). O número de espécies (Figura 6C) apresentou padrão oposto ao índice de Simpson, com medianas mais elevadas $(1,00)$ para as regiões de interior (IC e IM) do que as de borda (BM e BR) $\left(\chi^{2}=40,61, p=7,89 \times 10^{-9}, \mathrm{gl}=3\right)$. A região $\mathrm{IC}$ foi a única que diferiu em relação a todas as outras regiões considerando o índice de Pielou (Figura 6D), exceto com IM $\left(\chi^{2}=23,51, p=3,17 \times 10^{-5}, \mathrm{gl}=3\right)$. O estrato IC também apresentou diferença em relação aos demais em relação às densidades agregadas de todos os moluscos (Figura 6E) $\left(\chi^{2}=42,17, p=3,69 \times 10^{-9}, \mathrm{gl}=3\right.$ ) e nas densidades de bivalves (Figura $6 \mathrm{~F})\left(\chi^{2}=17,26\right.$, $p=6,23 \times 10^{-4}, \mathrm{gl}=3$ ). 
FIGURA 6: Diagramas de caixas com os índices de diversidade de Shannon (A), Simpson (B), Número de espécies (C), Pielou (D), Densidade de moluscos agregados (E), Densidade de bivalves agregados (F) da malacofauna capturada no banco extrativista da praia de Mangue Seco considerando as regiões borda mar (BM), borda rio (BR), interior costa (IC) e interior mar (IM).

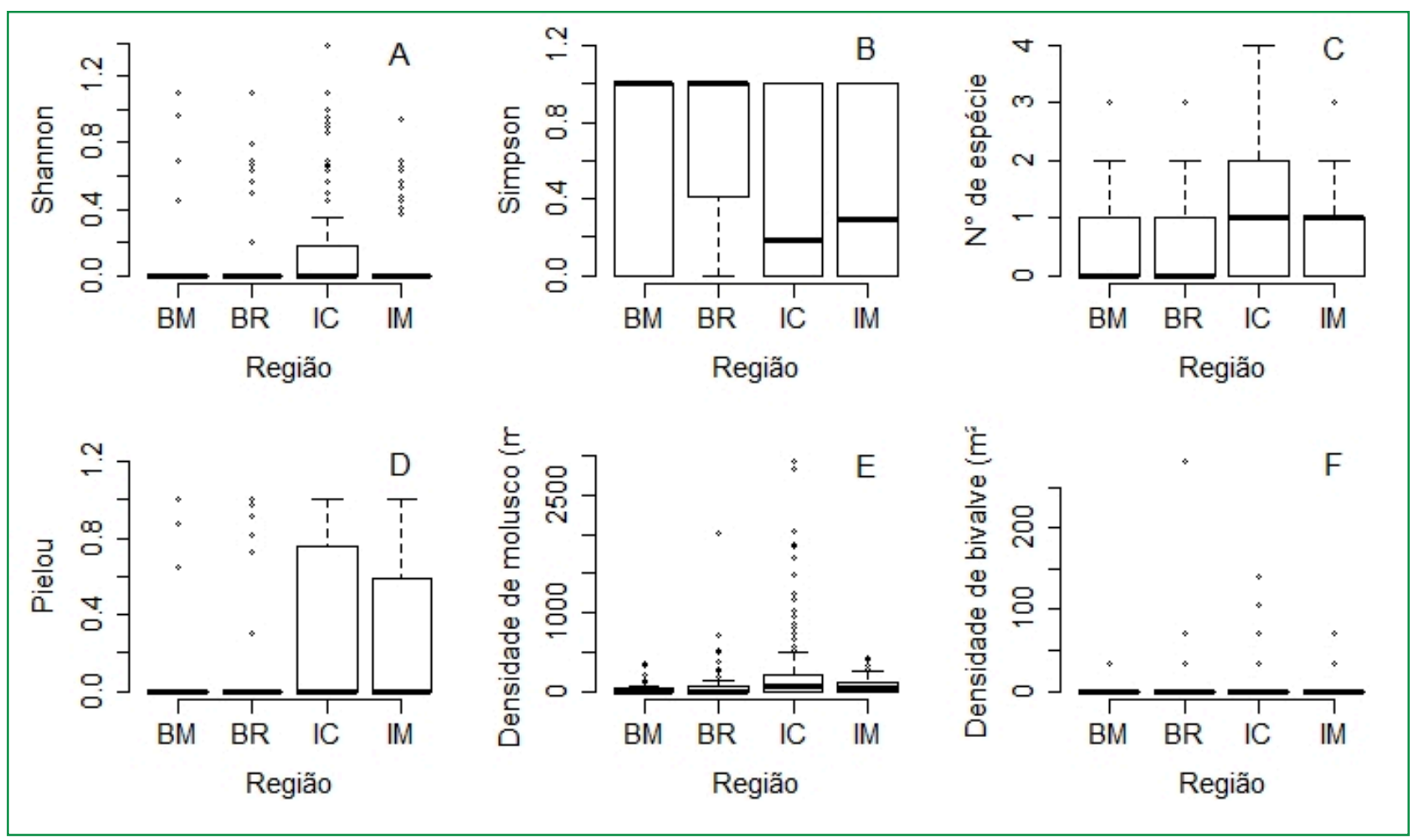

As correlações entre a densidade de $A$. flexuosa com todos os outros fatores (diversidades e densidades dos outros moluscos) não apresentaram valores elevados (Tabela 3), porém estatisticamente foram significativas $(p<0,05)$. Os maiores coeficientes de determinação apresentados com a densidade de A. flexuosa, de maneira decrescente, ocorreram entre essa variável com o índice de Pielou, o número de espécie, a densidade agregada de moluscos, o índice de Simpson, o índice de Shannon-Weaver e a densidade agregada de bivalves, respectivamente.

TABELA 3: Análise de correlação entre as densidades de Anomalocardia flexuosa com os índices de Shannon-Weaver, Simpson, Número de espécies, Pielou, Densidade de moluscos agregados, Densidade de bivalves agregados no banco extrativista da praia de Mangue Seco.

\begin{tabular}{lcccc}
\hline \multirow{2}{*}{ Variáveis correlacionadas } & \multicolumn{3}{c}{ Densidade de Anomalocardia flexuosa $\left.\mathbf{( m}^{\mathbf{2}}\right)$} \\
\cline { 2 - 5 } & Correlação $\left(\mathbf{R}^{2}\right)$ & Intercepto & Coeficiente angular & valor de $\mathbf{p}$ \\
\hline Shannon-Weaver & 0,34 & 240,2 & 359,0 & $2,69 \times 10^{-12}$ \\
Simpson & $-0,39$ & 394,1 & $-216,4$ & $2,20 \times 10^{-16}$ \\
Número de espécies & 0,50 & 162,8 & 150,7 & $2,20 \times 10^{-16}$ \\
Pielou & 0,52 & 171,2 & 380,9 & $2,20 \times 10^{-16}$ \\
Densidade de moluscos agregados $\left(\mathrm{m}^{2}\right)$ & 0,47 & 248,1 & 0,2 & $2,20 \times 10^{-16}$ \\
Densidade de bivalves agregados $\left(\mathrm{m}^{2}\right)$ & 0,30 & 246,8 & 4,1 & $4,59 \times 10^{-10}$ \\
\hline
\end{tabular}




\section{Discussão}

O Filo Mollusca é composto por oito Classes (Gastropoda, Bivalvia, Cephalopoda, Monoplacophora, Polyplacophora, Scaphopoda, Solenogaster e Caudofoveata), porém apenas as duas primeiras foram identificadas no banco. A classe Gastropoda tem maior diversidade de espécies mundial, ocupando ambientes marinhos, límnicos e terrestres, porém teve menor quantidade de espécies identificadas nesta praia arenosa tropical do que a classe Bivalvia, que geralmente corresponde a espécies de maior interesse na atividade de pesca. Além da $A$. flexuosa, que é uma das espécies de moluscos mais comercializadas e consumidas no Brasil (BOEHS et al., 2010), os bivalves I. brasiliensis e L. pectinata são importantes recursos pesqueiros (BEASLEY et al., 2005; SILVA et al., 2000). As conchas desses bivalves também são comercializadas como matéria prima para o zooartesanato, assim como os demais moluscos identificados (ALVES et al., 2006).

Apesar da curva de rarefação ter atingido uma assíntota, o número de espécies identificadas poderia ter sido maior, pois de acordo com os pescadores locais, a espécie bivalve Tagelus plebeius, por exemplo, era um importante recurso pesqueiro, mas se encontra bastante escasso. A presença dessa espécie no local foi também relatada por Moura (2009). Foram observadas conchas vazias do bivalve na área avaliada, porém, a ausência desse bivalve pode ser um indicador de que essa espécie, anteriormente explorada, teria sido afetada por fatores antrópicos (e.g. esforço de pesca) e, mesmo com o aumento do número de amostras, T. plebeius seria uma espécie difícil de ser encontrada atualmente.

Anomalocardia flexuosa é a principal espécie alvo da captura no banco de Mangue Seco, portanto há um grande número de pescadores que atuam na extração desses indivíduos, o que denota um esforço de pesca maior comparado a outras espécies da malacofauna. Esse esforço poderia afetar a sua frequência como consequência de uma sobre-exploração, porém os valores de densidade foram superiores a todos os outros moluscos (explorados ou não), além disso sua distribuição foi a mais extensa na área. As outras espécies de bivalves comercializadas para consumo, no entanto, não apresentaram grandes densidades e amplitude espacial. Nesta praia arenosa tropical, ocorreu um menor número de espécies identificadas do que na Península de Ajuruteua no estado do Pará, porém praticamente as mesmas espécies foram encontradas (BEASLEY et al., 2005). A principal diferença entre as regiões consistiu na discrepância em relação às distribuições e densidades durante os meses, pois na Península de Ajuruteua foi mais uniforme, o que pode sugerir que o ambiente seja mais dinâmico, sem a presença de um molusco dominante.

As distribuições dos indivíduos da macrofauna bentônica são afetadas por interações bióticas tanto em ambientes físicos estressantes (DUGAN et al., 2004) como em ambientes mais amenos (MATTOS; CARDOSO, 2012). As propriedades físicas são importantes para distribuição da macrofauna bentônica de regiões dissipativas que têm características mais amenas, como, por exemplo, menos intensidade de correntes e ondas, podendo propiciar maior oferta de alimento para as espécies da macrofauna bentônica que as regiões que apresentam características reflexivas, que sofrem pelas ações de ondas (DEFEO et al., 2001; MCLACHLAN; DORVLO, 2005). A região IC do banco tem características mais dissipativas por ser uma área abrigada em relação às demais, podendo ser um dos fatores de as espécies serem mais frequentes, principalmente para as espécies bivalves como $A$. flexuosa, que tem sifão curto e preferência por habitat com pouca ressuspensão de sedimentos (NARCHI, 1974; BOEHS et al., 2008).

Nos meses de coletas, as densidades de $A$. flexuosa variaram, assim como nos estratos de localização, porém há ausência de diferença entre os índices de diversidade e as densidades da malacofauna (agregados todos ou só bivalves) ao longo dos meses, indicando pouca variabilidade na composição dessas espécies e suas densidades. O banco de praia arenoso estudado foi considerado uma região de grande diversidade (OLIVEIRA et al., 2011), porém foi notório entre os moluscos o predomínio de duas espécies, o gastrópode $N$. virginea e o bivalve $A$. flexuosa. Há dominância de $A$. flexuosa também em outros locais, como, por exemplo, na praia de Flexeiras, localizada no Sudeste do Brasil. 
Neste local, a densidade média de $A$. flexuosa foi superior à encontrada para outras espécies abundantes, como Diplodonta punctata (MATTOS; CARDOSO, 2012) e as espécies $T$. lineata e $T$. versicolor (CARDOSO et al., 2015). Há exceções da presença de A. flexuosa com altas densidades (mas não como espécie de molusco dominante). Foi o caso de $N$. virginea, que apresentou maior abundância na praia da Barra na região (CRUZNETA; HENRY-SILVA, 2013).

As correlações significativas positivas entre as densidades de A. fleuxosa com as medidas de ShannonWeaver, número de espécie, Pielou e as densidades agregadas dos outros moluscos demonstram que, de maneira geral, há sobreposição dos moluscos, sendo que isso deve ocorrer principalmente na região IC do banco, pois esse estrato apresentou uma maior diversidade e densidade da malacofauna quando comparado aos demais. A correlação negativa com índice de Simpson também foi um indicativo de que a espécie teve preferência por região mais diversificada, pois com o aumento do valor desse índice menor seria a diversidade. Neste trabalho, a interação da A. flexuosa não foi avaliada considerando as espécies individualmente, porém, das medidas estimadas, a de Pielou foi a que teve maior coeficiente de determinação $\left(\mathrm{R}^{2}\right)$, ou seja, o aumento da uniformidade das outras espécies agregadas ofereceu uma maior confiabilidade para que também ocorresse um aumento das densidades da $A$. flexuosa. Portanto, um ambiente com maior equitabilidade entre as espécies pode ser um indicativo de condições propícias para o estabelecimento destas, logo, uma interação competitiva foi minimizada, pois não deve ocorrer, por exemplo, escassez de alimentos para nenhuma das espécies envolvidas.

Há grande sobreposição das espécies de moluscos nesta praia arenosa estudada, sendo a região interior costa (IC) a que teve maiores e mais diversificadas agregações. As distribuições e abundância da A. flexuosa como espécie dominante não foi afetada pelos demais moluscos e possivelmente não deve ocorrer o oposto, podendo a associação entre espécies não ser um fator determinante para controlar as densidades e frequência nesta praia arenosa. As tomadas de decisão para sustentabilidade da captura de $A$. flexuosa na área estudada devem também considerar todos os outros moluscos para mitigar futuros danos à diversidade da malacofauna na região, principalmente dessas espécies em que não há interesse econômico, mas que podem sofrer algum tipo de impacto devido à mobilização do sedimento com as artes de pesca utilizadas. Assim, estudos de seletividade dos meios de captura usados na atividade devem ser realizados para avaliar tamanhos de primeira captura que possibilitem, além da obtenção da A. flexuosa, o escape dos outros moluscos não alvo e, posteriormente, o comportamento desses indivíduos (taxa de mortalidade) após o contato e escape com o artefato.

\section{Agradecimentos}

À Coordenação de Aperfeiçoamento de Pessoal de Nível Superior (CAPES), por conceder uma bolsa de estudo de pós-graduação no momento da pesquisa ao primeiro autor.

\section{Referências}

ABBOTT, R. T. American seashell. 2. ed. New York: Van Nostrand, 1974. $663 \mathrm{p}$.

ALVES, M. S.; SILVA, M. A.; MELO JÚNIOR, M.; PARANAGUÁ, M. N.; PINTO, S. L. Zooartesanato comercializado em Recife, Pernambuco, Brasil. Revista Brasileira de Zoociências, Juiz de Fora, v. 8, n. 2, p. 99-109, 2006.

AMARAL, A. C. Z.; JABLONSKI, S. Conservation of marine and coastal biodiversity in Brazil. Conservation Biology, San Francisco, v. 19 , n. 3, p. 625-631, 2005.

BARROSO, C. X.; MATTHEWS-CASCON, H. Distribuição espacial e temporal da malacofauna no estuário do rio Ceará, Ceará, Brasil. Pan-American Journal of Aquatic Sciences, Rio Grande, v. 4, n. 1, p. 79-86, 2009.

BEASLEY, C. R.; FERNANDES, C. M.; GOMES, C. P.; BRITO, B. A.; SANTOS, S. M. L.; TAGLIARO, C. H. Molluscan diversity and abundance among coastal habitats of northern Brazil. Ecotropica, Frankfurt, v. 11, n. 1, p. 9-20, 2005.

BOEHS, G.; ABSHER, T. M.; CRUZ-KALED, A. C. Ecologia populacional de Anomalocardia brasiliana (Gmelin, 1791) (Bivalvia, Veneridae) na Baía de Paranaguá, Paraná, Brasil. Boletim do Instituto de Pesca, São Paulo, v. 34, n. 2, p. 259-270, 2008.

BOEHS, G.; MAGALHÃES, A. R. M. Simbiontes associados com Anomalocardia brasiliana (Gmelin) (Mollusca, Bivalvia, Veneridae) na Ilha de Santa Catarina e região continental adjacente, Santa Catarina, Brasil. Revista Brasileira de Zoologia, Curitiba, v. 21, n. 4, p. 865-869, 2004.

BOEHS, G.; VILLALBA, A.; CEUTA, L. O.; LUZ. J. R. Parasites of three commercially exploited bivalve mollusc species of the estuarine region of the Cachoeira River (Ilhéus, Bahia, Brazil). Journal of Invertebrate Pathology, Riverside, v. 103, n. 1, p. 43-47, 2010. 
BORZONE, C. A.; SOUZA, J. R. B.; SOARES, A. G. Morphodynamic influence on the structure of inter and subtidal macrofaunal communities of subtropical sandy beaches. Revista Chilena de História Natural, Santiago, v. 69, n. 4, p. 565-577, 1996. CARDOSO, R. S.; GALHARDO, L. M.; CABRINI, T. M. N. Population ecology and secondary production of congeneric bivalves on a sheltered beach in Southeastern Brazil. Journal of Shellfish Research, Washington, v. 34, n. 3, p. 931-938, 2015.

CRUZ-NETA, C. P.; HENRY-SILVA, G. G. Aspectos da dinâmica populacional do gastrópode Neritina virginea em região estuarina do Rio Grande do Norte, Brasil. Boletim do Instituto de Pesca, São Paulo, v. 39, n. 1, p. 1-14, 2013.

DEFEO, O.; GÓMEZ, J.; LERCARI, D. Testing the swash exclusion hypothesis in sandy beach populations: the mole crab Emerita brasiliensis in Uruguay. Marine Ecology Progress Series, Luhe, v. 212, n. 4, p. 159-170, 2001.

DEXTER, D. M. Sandy beach community structure: the role of exposure and latitude. Journal of Biogeography, New York, v. 19, n. 1, p. 59-66, 1992.

DUGAN, J.E.; JARAMILLO, E.;HUBBARD, D. M.; CONTRERAS, H.; DUARTE, C. Competitive interactions in macroinfaunal animals of exposed sandy beaches. Oecologia, Heidelberg, v. 139, n. 2, p. 630-640, 2004.

HALL, S. J. Physical disturbance and marine benthic communities: life in unconsolidated sediments. Oceanography and Marine Biology, Aberdeen, v. 32, n. 1, p. 179-239, 1994.

HENSEN, C.; ZABEL, M.; SCHULZ, H. N. Benthic cycling of Oxygen, Nitrogen and Phosphorus. In: SCHULZ, H. D.; ZABEL, M. (Ed.). Marine geochemistry. Berlin, Heidelberg: Springer, 2006. p. $207-240$.

JARAMILLO, E.; CONTRERAS, H.; DUARTE, C.; QUIJÓN, P. Relationships between community structure of the intertidal macroinfauna and sandy beaches characteristics along the Chilean coast. Marine Ecology, Berlin, v. 22, n. 4, p. 323-342, 2001.

LAVANDER, H. D.; CARDOSO JUNIOR, L. O.; OLIVEIRA, R. L.; SILVA NETO, S. R.; GÁLVEZ, A. O.; PEIXOTO, S. R. M. Biologia reprodutiva da Anomalocardia brasiliana (Gmelin, 1791) no litoral norte de Pernambuco, Brasil. Revista Brasileira de Ciências Agrárias, Recife, v. 6, n. 2, p. 344-350, 2011.

LIMA, S. A. O.; ANDRADE, H. A. Zoneamento da área extrativista mais produtiva da Anomalocardia flexuosa (Linnaeus, 1767) (Mollusca: Bivalvia) no mundo. Biotemas, Florianópolis, v. 31, n. 3, p. 33-41, 2018.

MATTOS, G.; CARDOSO, R. S. Population dynamics of two suspension-feeding bivalves on a sheltered beach in southeastern Brazil. Helgoland Marine Research, Berlin, v. 66, n. 3, p. 393-400, 2012.

MCLACHLAN, A.; BROWN, A. The ecology of sandy shores. 2. ed. New York: Academic Press, 2006. 392 p.

MCLACHLAN, A.; DORVLO, A. Global patterns in sandy beach macrobenthic communities. Journal of Coastal Research, Fort Lauderdale, v. 21, n. 4, p. 674-687, 2005.

MICHAUD, E.; DESROSIERS, G.; MERMILLOD-BLONDIN, F.; SUNDBY, B.; STORA, G. The functional group approach to bioturbation: I, The effects of biodiffusers and gallery-diffusers of the Macoma balthica community on sediment oxygen uptake. Journal of Experimental Marine Biology and Ecology, Amsterdam, v. 326, n. 1, p. 77-88, 2005

MICHAUD, E.; DESROSIERS, G.; MERMILLOD-BLONDIN, F.; SUNDBY, B.; STORA, G. The functional group approach to bioturbation: II, The effects of the Macoma balthica community on fluxes of nutrients and dissolved organic carbon across the sedimentwater interface. Journal of Experimental Marine Biology and Ecology, Amsterdam, v. 337, n. 2, p. 178-189, 2006.

MOURA, R. T. Aspectos gerais da hidrobiologia do litoral norte de Pernambuco - Brasil. Brasília: IBAMA - MMA, 2009. 138 p.

NARCHI, W. Aspectos ecológicos e adaptativos de alguns bivalves do litoral paulista. Papéis Avulsos Zoologia, São Paulo, v. 27, p. 235-262, 1974.

OKSANEN, J.; BLANCHET, F. G.; FRIENDLY, M.; KINDT, R.; LEGENDRE, P.; MCGLINN, D.; MINCHIN, P. R.; O'HARA, R. B.; SIMPSON, G. L.; SOLYMOS, P.; STEVENS, M. H. H.; SZOECS, E.; WAGNER, H. Vegan: Community Ecology Package. 2018. Disponível em: $<$ https:// CRAN.R-project.org/package=vegan/>.

OLIVEIRA, I. B.; AMORIM, A.; LAVANDER, H.; PEIXOTO, S.; GÁLVEZ, A. O. Spatial and temporal distribution of the shellfish Anomalocardia brasiliana (Gmelin, 1791) on Mangue Seco beach, Pernambuco, Brazil. International Journal of Aquatic Science, Urmia, v. 2, n. 1, p. 68-79, 2011

OLIVEIRA, I. B.; SILVA NETO, S. R.; LIMA FILHO, J. V. M.; PEIXOTO, S. R. M.; GALVEZ, A. O. Efeito do período chuvoso na extração do molusco bivalve Anomalocardia brasiliana (Gmelin, 1791). Revista Brasileira de Ciências Agrárias, Recife, v. 9, n. 1, p. 139-145, 2014.

PEZZUTO, P. R.; ECHTERNACHT, A. M. Avaliação de impactos da construção da Via Expressa SC-Sul sobre o berbigão Anomalocardia brasiliana (Gmelin, 1791) (Mollusca: Pelecypoda) na Reserva Extrativista Marinha do Pirajubaé (Florianópolis, Brasil). Atlântica, Rio Grande, v. 21, n. 1, p. 105-119, 1999.

PIELOU, E. C. An introduction to Mathematical Ecology. New York: Wiley Interscience, 1969. 286 p.

R CORE TEAM. R: a language and environment for statistical computing. 2018. Disponível em: <https://www.R-project.org/>.

RIOS, E. C. Seashells of Brazil. 2. ed. Rio Grande: Fundação da Universidade do Rio Grande, 1994. 492 p.

SCHAEFFER-NOVELLI, Y.Análise populacional de Anomalocardia brasiliana (Gmelin, 1791) (Mollusca: Bivalvia), na praia do Saco da Ribeira, Ubatuba, Estado de São Paulo. Boletim do Instituto de Oceanografia, São Paulo, v. 29, n. 2, p. 351-35, 1980.

SHANNON, C. E.; WEAVER, W. The Mathematical Theory of Communication. Urbana: University of Illinois Press, 1949. $131 \mathrm{p}$.

SILVA, G. S.; MELLO, R. L. S.; NASCIMENTO, A. E.; MESSIAS, A. S.; ARAÚJO, S. F. S. As atividades pesqueiras artesanais e a relação com a malacofauna no manguezal do Rio Formoso, PE Brasil. Trabalhos Oceanográficos da Universidade Federal de Pernambuco, Recife, v. 28, n. 2, p. 195-207, 2000.

SIMPSON, E. H. Measurement of diversity. Nature, New York, v. 163, n. 4148, p. $688,1949$.

WARMKE, G. L.; ABBOTT, R. T. Caribbean seashells: a guide to the marine mollusks of Puerto Rico and other West Indian islands, Bermuda and the Lower Florida Keys. Narberth: Livingston Publishing Company, 1961. 346 p. 